Agrotrópica 32(3): 225 - 232. 2020.

Centro de Pesquisas do Cacau, Ilhéus, Bahia, Brasil

\title{
REFRIGERAÇÃO NA CONSERVAÇÃO DE FLORES DE CAPUCHINHA
}

\author{
Alexandra Goede de Souza, Jaqueline Carvalho, Jéssica Mayumi Anami, Eduardo Affonso \\ Jung, Paula Hamerski \\ Instituto Federal Catarinense - Campus Rio do Sul, Estrada do Redentor, 5665, Canta Galo, 89163-356, Rio do Sul, Santa \\ Catarina, Brasil. \\ alexandra.souza@ifc.edu.br;jaquelinecarvalho1991@gmail.com; mayumi_anami@yahoo.com.br; \\ eduardojung2000@outlook.com; paula.hamerski@ifc.edu.br
}

\begin{abstract}
O objetivo do trabalho foi avaliar a conservação pós-colheita de flores de capuchinha armazenadas em diferentes temperaturas do ar. Após a colheita, as flores foram acondicionadas em embalagens de polietileno tereftalato (PET) e submetidas aos tratamentos: armazenamento em temperatura ambiente $\left(22 \pm 2^{\circ} \mathrm{C}\right.$ e umidade relativa (UR) de $75 \% \pm 5 \%)$ e refrigerada $\left(5 \pm 1^{\circ} \mathrm{C}, 10 \pm 1^{\circ} \mathrm{C}\right.$ e $15 \pm 1^{\circ} \mathrm{C}$ e UR de $\left.90 \pm 5 \%\right)$. Na colheita e após armazenamento final de cada tratamento, foram avaliadas a perda de massa fresca (MF), teor de sólidos solúveis (SS), acidez total titulável (AT), relação SS/AT, pH, conteúdo de vitamina $\mathrm{C}$, além de murcha e escurecimento aparente das flores. As maiores perdas de MF, maior murcha e escurecimento aparente, menores teores de SS, AT, relação SS/AT e conteúdo de vitamina C ocorreram no tratamento com armazenamento em temperatura do ar ambiente. $\mathrm{O}$ armazenamento refrigerado a $5^{\circ} \mathrm{C} \mathrm{e}$ $10^{\circ} \mathrm{C}$ promoveu maior conteúdo de vitamina $\mathrm{C}$ e menor murcha e escurecimento aparente do tecido, mantendo as flores de capuchinha aptas para o consumo por mais tempo.
\end{abstract}

Palavras-chave: flores comestíveis, plantas alimentícias não convencionais, Tropaeolum majus L., vitamina C.

Refrigeration in the conservation of nasturtium flowers. The objective of this work was to evaluate the postharvest conservation of nasturtium flowers stored at different air temperatures. After harvesting, the flowers were placed in polyethylene terephthalate (PET) packaging and submitted to treatments: storage at room temperature $\left(22 \pm 2^{\circ} \mathrm{C}\right.$ and relative humidity $(\mathrm{RH})$ of $\left.75 \% \pm 5 \%\right)$ and refrigerated $\left(5 \pm 1{ }^{\circ} \mathrm{C}, 10 \pm 1{ }^{\circ} \mathrm{C}\right.$ and $15 \pm 1{ }^{\circ} \mathrm{C}$ and $\mathrm{RH}$ of $90 \pm 5 \%$ ). At harvest and after storage in each treatment, the fresh weight loss, soluble solids (SS), total titratable acidity (TA), SS/TA ratio, $\mathrm{pH}$, vitamin $\mathrm{C}$ content and apparent wilting and darkening in the flowers were evaluated. The highest MF loss, higher apparent wilting and darkening, lowest SS, TA, SS/TA ratio and vitamin C content occurred in the treatment with storage at room air temperature. Cold storage at $5^{\circ} \mathrm{C}$ and $10^{\circ} \mathrm{C}$ promoted highest vitamin $\mathrm{C}$ content and lowest apparent wilting and darkening, keeping the nasturtium flowers suitable for consumption for more time.

Key words: edible flowers, unconventional food plants, Tropaeolum majus L., vitamin C. 


\section{Introdução}

Apesar da utilização de flores na alimentação humana, no Brasil, estar restrita a espécies de couveflor e brócolis, está ocorrendo um aumento da utilização de outras flores comestíveis, especialmente pela presença de compostos bioativos benéficos à saúde (Kaisoon at al., 2011). Dentre as flores, cujo aumento do consumo vem crescendo, destaca-se a capuchinha (Tropaeolum majus L.), uma Planta Alimentícia Não Convencional (PANC), caracterizada por não ser utilizada regularmente na alimentação (Kelen et al., 2015). A capuchinha, popularmente conhecida também como nastúrcio, alcaparra-de-pobre, entre outros, tem o Peru e o México como centro de origem primária (Lorenzi e Matos, 2007), mas atualmente é encontrada em várias partes do mundo, incluindo o Brasil. Trata-se de uma liana de hábito herbáceo, com folhas grandes, simples de aspecto circular e flores de cores vibrantes em formato de campânula (Souza e Lorenzi, 2005).

Atualmente, a capuchinha é valorizada pelos restaurantes finos, que servem as flores em saladas, guarnições e doces nutritivos e atraentes, além da decoração de pratos. Devido ao seu sabor picante, as flores de capuchinha tem grande aceitação na alimentação, aliando aspectos organolépticos e nutracêuticos (Benvenuti, Bortolotti \& Maggini, 2016). Essas plantas tem ação anticoagulante (Santo et al., 2007), antiescorbútica, hipertensora, anti-inflamatória, calmante, expectorante e digestiva. Também são fonte de vitamina $\mathrm{C}$, antocianinas, carotenoides, compostos fenólicos e apresentam alta atividade antioxidante (Liberato, Lima e Silva, 2019).

Em geral, as flores são consideradas de alta perecibilidade, necessitando de condições adequadas para manter por mais tempo sua coloração, frescor e longevidade pós-colheita. A utilização do armazenamento refrigerado, é uma técnica empregada com sucesso em pós-colheita de muitos vegetais íntegros (Freddo, Cechin \& Mazaro, 2013) ou minimamente processados (Souza et al., 2019), permitindo o transporte a longas distâncias e o armazenamento prolongado com reduzida perda de qualidade. A temperatura de armazenamento é o fator ambiental mais importante, tanto do ponto de vista comercial como do controle da senescência por regular diretamente as taxas de todos os processos fisiológicos e bioquímicos dos vegetais (Chitarra e Chitarra 2005). Baixas temperaturas reduzem a produção de etileno, a respiração, retardam a degradação das reservas de açúcares ou outros substratos, prolongando a durabilidade das flores (Sonego e Brackmann, 1995).

Diante à escassez de trabalhos científicos sobre a conservação pós-colheita de flores de capuchinha, este trabalho tem como objetivo avaliar os atributos físicos e químicos em flores de capuchinha submetidas ao armazenamento refrigerado.

\section{Material e Métodos}

As plantas de capuchinha 'Jewel' de cor laranja foram produzidas na área experimental do Instituto Federal Catarinense (IFC) - Campus Rio do Sul, SC. As mudas foram produzidas em bandejas de isopor com substrato comercial orgânico e transplantadas após 30 dias da emergência para quatro canteiros com área de $2 \mathrm{~m}^{2}$ cada. Nos canteiros foram aplicados 22.000 $\mathrm{kg} / \mathrm{ha}$ de esterco de peru e incorporados com auxílio de um encanteirador quinze dias antes do transplante. A colheita foi realizada manualmente no início da manhã e imediatamente transportadas ao Laboratório de Fisiologia Vegetal e Pós-colheita local. Foram colhidas somente as flores que estavam no estádio XII, caracterizada pela antese total (Silva et al., 2018).

As flores foram classificadas, sendo retiradas aquelas com defeitos enquanto as demais foram acondicionadas em embalagens de polietileno tereftalato (PET) nas dimensões $217 \mathrm{~mm}$ x $147 \mathrm{~mm} \mathrm{x}$ $68 \mathrm{~mm}$. As embalagens contendo as flores foram armazenadas nas temperaturas do ar de $5 \pm 1^{\circ} \mathrm{C}$ e UR de $90 \pm 5 \% ; 10 \pm 1^{\circ} \mathrm{C}$ e UR de $90 \pm 5 \% ; 15 \pm 1{ }^{\circ} \mathrm{C}$ e UR de $90 \pm 5 \%$ (em incubadora B.O.D. com controle de temperatura e umidade, sem luz); e em temperatura do ar ambiente $\left(22 \pm 2{ }^{\circ} \mathrm{C}\right.$ e UR de $\left.75 \% \pm 5 \%\right)$ por oito dias. A testemunha foi representada pelas análises realizadas no momento da colheita para efeitos de comparação.

Na colheita e após oito dias do armazenamento das flores foram avaliados a perda de massa fresca (MF), a murcha e o escurecimento aparente, teor de sólidos solúveis (SS), acidez total titulável (AT), relação SS/ AT, pH e conteúdo de vitamina C. Nas flores de capuchinha armazenadas sem refrigeração, todas as 
análises foram realizadas após seis dias de armazenamento, pois as flores apresentavam sinais avançados de senescência.

Para avaliação da perda de massa fresca total foram realizadas pesagens das flores com balança analítica a cada dois dias, sendo: 0 (dia da colheita), 2 , 4, 6 e 8 dias de armazenamento.

A murcha e o escurecimento aparente das flores de capuchinha foram avaliados utilizando-se uma escala evolutiva (notas) de acordo com Souza et al. (2016) com adaptações. A escala varia de 1 a 4, sendo: 1) $<25 \%$ de escurecimento ou murcha; 2) $26-50 \%$ de escurecimento ou murcha; 3 ) $51-75 \%$ de escurecimento ou murcha e 4) $76-100 \%$ de escurecimento ou murcha do tecido.

Os teores de AT foram obtidos por titulometria de $10 \mathrm{~mL}$ do suco das flores (processada com auxílio de um "mixer") diluídos em $90 \mathrm{~mL}$ de água destilada com hidróxido de sódio $0,1 \mathrm{~N}$ até o ponto de viragem (Instituto Adolfo Lutz, 2008) e os resultados expressos em porcentagem de ácido cítrico. Os teores SS foram determinados em refratômetro digital com compensação automática de temperatura em suco extraído das flores conforme descrito para AT. A relação entre SS/AT foi calculada pela divisão dos SS pela AT. O pH foi determinado com pHmetro de bancada (modelo mPA210) também com suco extraído das flores (Instituto Adolfo Lutz, 2008).

$\mathrm{O}$ conteúdo de vitamina $\mathrm{C}$ foi determinado pelo método espectrofotométrico, utilizando-se 2,4-denitrofenilhidrazina (Amarante et al., 2019). Foi utilizado $1 \mathrm{~g}$ da amostra, macerado em 50 $\mathrm{mL}$ de ácido oxálico $(0,5 \%)$. Após filtragem, em $1 \mathrm{~mL}$ da amostra foram adicionados $3 \mathrm{~mL}$ de ácido oxálico, cinco gotas do agente oxidante 2,6 diclorofenol-indofenol (2,6-DCFI), $1 \mathrm{~mL}$ de 2,4 dinitrofenilhidrazina (2,4-DNPH), uma gota de tiouréia e $5 \mathrm{~mL}$ de ácido sulfúrico. As leituras foram realizadas em espectrofotômetro (Kasiv - K37-Vis) a $520 \mathrm{~nm}$, e os resultados expressos em mg de ácido ascórbico $100 \mathrm{~g}^{-1}$ de MF.

O delineamento estatístico foi inteiramente casualizado com cinco tratamentos e quatro repetições, cada repetição composta por três embalagens com 20 flores. Os dados foram testados para os pressupostos da análise variância usando Bartlett (teste de homogeneidade de variância) e Shapiro-Wilk (teste de normalidade). Atendidos os requisitos os dados não transformados foram submetidos à análise de variância e comparados pelo teste de Tukey a $5 \%$ de probabilidade utilizando-se o programa SASM-Agri (Canteri et al., 2001).

\section{Resultados e Discussão}

A perda de massa fresca total das flores de capuchinha foi maior no tratamento com armazenamento em temperatura ambiente, seguido pelo armazenamento refrigerado a $15^{\circ} \mathrm{C}$, enquanto para os demais tratamentos as perdas não apresentaram diferenças significativas. Após seis dias do armazenamento em temperatura ambiente a perda média de massa fresca foi de $43,2 \%$, enquanto no armazenamento refrigerado a $15^{\circ} \mathrm{C}, 10^{\circ} \mathrm{C}$ e $5^{\circ} \mathrm{C}$ a perda foi de $30,2 \%, 21,8 \%$ e $21,7 \%$, respectivamente, após oito dias do armazenamento (Figura 1). A maior perda no armazenamento sem refrigeração pode ser decorrente da maior transpiração, provocada pelas altas temperaturas, resultando na desidratação dos tecidos (Freddo, Cechin \& Mazaro, 2013), indicando que o armazenamento a temperatura de $15^{\circ} \mathrm{C}$ pode ser considerada alta para conservação das flores desta espécie. Segundo Sonego e Brackmann (1995), o armazenamento de flores de rosa por um dia na

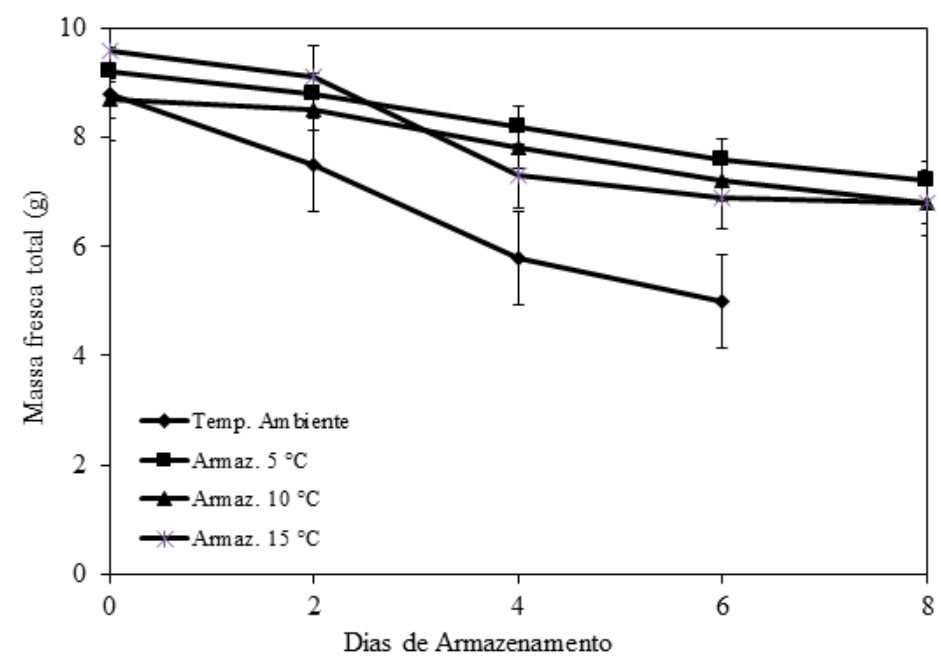

Figura 1. Perda de massa fresca total em flores de capuchinha (Tropaeolum majus) durante o armazenamento.

As barras indicam diferenças significativas mínimas calculadas pelo teste de Tukey a 5\% de probabilidade. 
temperatura de $15^{\circ} \mathrm{C}$ equivale a três dias de armazenamento a $5^{\circ} \mathrm{C}$.

Os resultados deste estudo indicam que a refrigeração a $5^{\circ} \mathrm{C}$ ou $10^{\circ} \mathrm{C}$ pode auxiliar na redução da perda de massa fresca em flores de capuchinha durante o armazenamento por até oito dias. Resultados semelhantes foram encontrados em folhas de cebolinha-verde e couve-manteiga minimamente processadas onde o armazenamento refrigerado a $10^{\circ} \mathrm{C}$ promoveu perdas de $6,8 \%$ e $5,5 \%$, respectivamente, e de $60,5 \% 30 \%$ quando armazenado sem refrigeração (Souza, Carvalho e Anami, 2018a; Souza, Carvalho e Anami, 2018b). Para flores de capuchinha acondicionadas em bandejas de isopor e recobertas com filme de PVC armazenados a $5^{\circ} \mathrm{C}$ por oito dias foram reportadas perdas inferiores $(13,6 \%)$ as obtidas neste estudo (Sangalli, Scalon e Carvalho, 2007). As maiores perdas reportadas no presente estudo podem ser decorrentes das diferenças de embalagens utilizadas para o armazenamento. Mesmo as flores de capuchinha que permaneceram no campo após o ponto ideal de colheita (estádio XII) quantificou-se perdas de massa fresca, resultante do processo natural de senescência (Silva et al., 2018).

A refrigeração promoveu redução de murcha e do escurecimento aparente dos tecidos das flores de capuchinha, enquanto as flores submetidas ao armazenamento em temperatura do ar ambiente apresentaram os piores valores na escala evolutiva de murcha $(4,0)$ e escurecimento $(4,0)$ aparente (Tabela 1).

Tabela 1. Murcha e escurecimento aparente em flores de capuchinha (Tropaeolum majus) após o período final de armazenamento $^{1}$

\begin{tabular}{lcc}
\hline Tratamento & Murcha & Escurecimento \\
\hline Colheita (testemunha) & $1,0 \mathrm{c}^{2}$ & $1,0 \mathrm{c}$ \\
Armazenamento a $5{ }^{\circ} \mathrm{C}$ & $1,1 \mathrm{c}$ & $1,2 \mathrm{c}$ \\
Armazenamento a $10^{\circ} \mathrm{C}$ & $1,4 \mathrm{c}$ & $1,5 \mathrm{c}$ \\
Armazenamento a $15^{\circ} \mathrm{C}$ & $2,1 \mathrm{~b}$ & $3,2 \mathrm{~b}$ \\
Armazenamento Temp Ambiente & $4,0 \mathrm{a}$ & $4,0 \mathrm{a}$ \\
\hline Média & 1,9 & 2,2 \\
$\mathrm{CV}(\%)$ & 9,6 & 12,1 \\
\hline
\end{tabular}

${ }^{1}$ Escala de murcha e escurecimento: $1:<25 \% ; 2$ : entre $26-$ $50 \%$; 3 : entre $51-75 \%$; e 4 : entre $76-100 \%$ de murcha e escurecimento aparente.

${ }^{2}$ Valores seguidos pela mesma letra, na coluna, não diferem entre si pelo teste de Tukey $(\mathrm{p}<0,05)$.
A menor murcha foi observada nos tratamentos com armazenamento refrigerado a $5^{\circ} \mathrm{C}$ e $10^{\circ} \mathrm{C}$, os quais não diferiram estatisticamente da colheita, seguido do armazenamento refrigerado a $15^{\circ} \mathrm{C}$, corroborando com os resultados de perda de massa fresca das flores. Após seis dias de armazenamento, as flores de capuchinha armazenadas sem refrigeração estavam totalmente impróprias para o consumo, enquanto as demais permaneceram aptas até o oitavo dia, indicando que a murcha pode ser reduzida com o armazenamento refrigerado. Em folhas de cebolinha-verde e de couve manteiga minimamente processadas armazenadas sob refrigeração $\left(10^{\circ} \mathrm{C}\right)$ também apresentaram menor murcha quando comparado ao armazenamento em temperatura ambiente (Souza, Carvalho e Anami, 2018a; Souza, Carvalho e Anami, 2018b).

O escurecimento do tecido das flores de capuchinha também foi menor $(1,0)$ com o armazenamento refrigerado a $5^{\circ} \mathrm{C}$ e $10^{\circ} \mathrm{C}$, não diferindo do momento da colheita (Tabela 1). O escurecimento promove alterações indesejáveis na aparência e na qualidade sensorial do produto, reduzindo a vida útil e o valor comercial, especialmente produtos com vida de prateleira reduzida. O escurecimento pode ser provocado por a uma série de fatores, como estresses ou a presença de fenóis que, quando oxidados pelas enzimas polifenóis oxidases (PPOs), resultam no escurecimento dos tecidos vegetais (Pietro et al., 2012). O armazenamento em temperaturas elevadas, pode provocar o colapso das células, favorecendo a atuação destas enzimas sobre os substratos, resultando no escurecimento do tecido. Por outro lado, a refrigeração reduz a atividade respiratória, mantendo por mais tempo a integridade da membrana plasmática, evitando o extravasamento de eletrólitos (Cherut, 2009), promovendo efeito positivo no retardo do escurecimento das flores de capuchinha.

Houve redução nos teores de SS de $26,8 \%$ e $68 \%$ nas flores de capuchinha armazenadas a $15^{\circ} \mathrm{C}$ e em temperatura ambiente, respectivamente, quando comparadas com o tratamento testemunha (Tabela 2). A redução dos teores de SS em temperaturas elevadas é esperada, pois nesse caso ocorre aumento da velocidade respiratória e maior utilização dos açúcares durante o armazenamento (Chitarra e Chitarra, 2005). Comportamento semelhante foi reportado em frutos de carambolas e folhas de cebolinha-verde 
Tabela 2. Sólidos solúveis (SS; \%), acidez titulável total (AT; \%), relação $\mathrm{SS} / \mathrm{AT}, \mathrm{pH}$ e conteúdo de vitamina $\mathrm{C}$ (Vit C; mg $100^{-1}$ de MF) em flores de capuchinha (Tropaeolum majus) após o período final de armazenamento

\begin{tabular}{lccccc}
\hline \multicolumn{1}{c}{ Adubação } & $\begin{array}{c}\mathrm{SS} \\
(\%)\end{array}$ & $\begin{array}{c}\text { AT } \\
(\%)\end{array}$ & SS/AT & $\mathrm{pH}$ & $\begin{array}{c}\text { Vit C } \\
\mathrm{mg} 100^{-1}\end{array}$ \\
\hline Colheita (testemunha) & $9,7 \mathrm{a}^{1}$ & $0,20 \mathrm{a}$ & $48,5 \mathrm{a}$ & $5,3 \mathrm{~b}$ & $80,2 \mathrm{a}$ \\
Armazenamento a $5^{\circ} \mathrm{C}$ & $9,6 \mathrm{a}$ & $0,21 \mathrm{a}$ & $45,7 \mathrm{a}$ & $5,3 \mathrm{~b}$ & $72,2 \mathrm{a}$ \\
Armazenamento a $10^{\circ} \mathrm{C}$ & $10,9 \mathrm{a}$ & $0,23 \mathrm{a}$ & $47,4 \mathrm{a}$ & $5,5 \mathrm{~b}$ & $69,9 \mathrm{a}$ \\
Armazenamento a $15^{\circ} \mathrm{C}$ & $7,1 \mathrm{~b}$ & $0,18 \mathrm{a}$ & $39,4 \mathrm{~b}$ & $5,5 \mathrm{~b}$ & $54,9 \mathrm{~b}$ \\
Armaz. temp. ambiente & $3,1 \mathrm{c}$ & $0,12 \mathrm{~b}$ & $25,8 \mathrm{c}$ & $6,3 \mathrm{a}$ & $46,8 \mathrm{~b}$ \\
\hline Média & 7,5 & 0,19 & 41,4 & 5,6 & 64,8 \\
$\mathrm{CV}(\%)$ & 12,3 & 11,2 & 14,2 & 2,5 & 10,2 \\
\hline
\end{tabular}

${ }^{1}$ Valores seguidos pela mesma letra, nas colunas, não diferem entre si pelo teste de Tukey $(\mathrm{p}<0,05)$.

minimamente processadas durante $\mathrm{o}$ armazenamento sem refrigeração (Ogassavara et al., 2009). Em capuchinha, cujas flores permaneceram no campo até a completa senescência, também foram observadas reduções no teor de SS a partir do ponto ideal de colheita (Silva et al., 2018). No entanto, quando as flores foram armazenadas a $5^{\circ} \mathrm{C}$ e $10^{\circ} \mathrm{C}$ por oito dias, os teores de SS não diferiram da testemunha (Tabela 2), indicando que para esta planta, o armazenamento refrigerado nessas temperaturas não afeta a qualidade deste atributo.

$\mathrm{O}$ valor médio de SS encontrado na colheita para as flores de capuchinha foi de $9,7 \%$, valor superior aos $6,0 \%, 5,6 \%$ e $7,8 \%$ reportados por outros autores para a espécie (Costa, Ribeiro e Barbosa, 2014; Ribeiro, Barbosa e Costa, 2012; Silva et al., 2018) e aos $5,6 \%, 4,56 \%$ e $5,5, \%$ para flores comestíveis de hemerocalis (Hemerocallis x hybrida), cravina (Dianthus chinensis) e boca-de-leão (Antirrhinum majus), respectivamente (Stefaniak \& Grzeszczuk, 2019). Porém, os valores são inferiores aos de outras flores comestíveis como de goiabeira- serrana (Acca sellowiana) (11,75\%) (Amarante et al., 2019) e opuntia (Opuntia stricta) (14,9\%) (Ammar et al., 2014).

Os teores de AT não diferiram da testemunha em nenhum dos tratamentos com armazenamento refrigerado. No entanto, quando armazenado em temperatura ambiente as flores de capuchinha apresentaram reduções de $40 \%$ na AT em relação à colheita (Tabela 2). Assim como para SS, o armazenamento em temperatura ambiente promoveu maior degradação dos ácidos orgânicos, acelerando o processo de senescência das flores. Reduções nos valores de AT também foram reportados para folhas de couve manteiga minimamente processadas quando armazenados em temperatura ambiente (Souza, Carvalho e Anami, 2018b).

Os valores médios de AT $(0,19 \%)$ são semelhantes aos reportados em flores de capuchinha laranja produzidas na Paraíba (Costa, Ribeiro e Barbosa, 2014), podendo ser considerada uma hortaliça de baixa acidez. Acidez elevada decorre da presença de ácidos orgânicos que contribuem na manutenção da qualidade pós-colheita dos tecidos vegetais por servir de fonte de oxidação no processo respiratório, indicando

que para flores de capuchinha, o armazenamento refrigerado é importante para manutenção da AT e conservação do produto. Além disso, vegetais com menores valores de AT apresentam maior aceitação pelo consumidor (Chitarra e Chitarra, 2005). Em flores de hemerocalis, cravina e boca-de-leão foram reportados AT de 0,31\%, 0,35\% e 0,29\%, respectivamente (Stefaniak \& Grzeszczuk, 2019), valores superiores aos encontrados para capuchinha neste estudo.

Assim como para SS e AT, os menores valores da relação SS/AT foram encontrados no armazenamento sem refrigeração $(25,8)$ seguido do armazenamento refrigerado a $15^{\circ} \mathrm{C}(39,4)$. As maiores relações foram obtidas na colheita $(48,5)$ e refrigeradas a $10^{\circ} \mathrm{C}(47,4)$ e $5^{\circ} \mathrm{C}(45,7)$, os quais não diferiram entre si (Tabela 2). Relações de SS/AT elevadas são decorrentes dos maiores valores de SS apresentados pelas flores de capuchinha na colheita e no armazenamento refrigerado nas menores temperaturas. Relação entre SS/AT de 42 foram reportados para flores de capuchinhas laranjadas produzidas na Paraíba (Costa, Ribeiro e Barbosa, 2014), semelhante a relação apresentada no tratamento da colheita do presente estudo. As alterações nos teores de açúcares e compostos ácidos podem promover alterações nas propriedades sensoriais do produto, especialmente na sensação de doçura e acidez (Barret, Beaulieu \& Shewfelt, 2010), sendo a relação entre SS/AT um indicador das qualidades organolépticas dos alimentos. Em flores de hemerocalis, cravina e boca-de-leão foram reportados SS/AT de 18,08, 16,08 e 17,99, respectivamente (Stefaniak \& Grzeszczuk, 2019), indicando serem flores de sabor menos agradável quando comparadas com a capuchinha. 
Os valores de $\mathrm{pH}$ não diferiram da testemunha em nenhum dos tratamentos com armazenamento refrigerado. No entanto, quando armazenado em temperatura ambiente os valores de $\mathrm{pH}$ aumentaram significativamente (Tabela 2). $\mathrm{O}$ aumento do $\mathrm{pH}$ pode ser atribuído à redução da AT quando do armazenamento não refrigerado, decorrente da oxidação dos ácidos orgânicos no processo respiratório. Os valores de $\mathrm{pH}$ das flores de capuchinha, na colheita, obtidos neste estudo $(5,3)$ foram semelhantes aos 5,7 reportados em flores de capuchinha produzidos na Paraíba (Ribeiro, Barbosa e Costa, 2012).

Houve redução do conteúdo de vitamina $\mathrm{C}$ nas flores de capuchinha armazenadas em temperatura ambiente e refrigeradas a $15^{\circ} \mathrm{C}$, não diferindo entre si, quando comparado com a colheita. Entretanto, as flores refrigeradas a $5^{\circ} \mathrm{C}$ e $10^{\circ} \mathrm{C}$ não diferiram significativamente da colheita (Tabela 2). Reduções nos conteúdos de vitamina $\mathrm{C}$ são comuns durante o armazenamento da maioria dos vegetais em função da oxidação enzimática do ácido ascórbico (Lee \& Kader, 2000). Por exemplo, morangos armazenados por três dias e fisális por 28 dias apresentaram, respectivamente, redução de 3,2\% e 74,1\% no conteúdo de vitamina C (Andrade Junior et al., 2016; Silva et al., 2013).

O conteúdo médio de vitamina $\mathrm{C}$ foi de $64,8 \mathrm{mg}$ $100 \mathrm{~g}^{-1}$ de MF, variando de 46,8 a 80,2 mg $100 \mathrm{~g}^{-1}$ de $\mathrm{MF}$, com menores valores no armazenamento em temperatura ambiente e maiores na colheita. $\mathrm{O}$ conteúdo de vitamina $\mathrm{C}$ presente nas flores avaliadas na colheita e refrigeradas a $5^{\circ} \mathrm{C}$ e $10^{\circ} \mathrm{C}$ são semelhantes e até superiores a frutos considerados fontes importantes desta vitamina, como a laranja (Citrus cinensis) (62,5 mg $\left.100 \mathrm{~g} \mathrm{~g}^{-1} \mathrm{de} \mathrm{MF}\right)$, goiaba (Psidium guajava) $\left(85,9 \mathrm{mg} 100 \mathrm{~g}^{-1} \mathrm{de} \mathrm{MF}\right)$ (Oliveira et al., 2011) e mamão (Carica papaya) (80,2 mg 100 $\mathrm{g}^{-1}$ de MF) (Couto e Canniatti-Brazaca, 2010). Para algumas flores também são reportados valores elevados de vitamina C como em amor-perfeito (Viola $x$ wittrockiana) (256 mg $100 \mathrm{~g}^{-1}$ de MF), capuchinha vermelha (Tropaeolum majus) (129 e 60,9 mg $100 \mathrm{~g}^{-1}$ de MF), cebolinha-verde (Allium schoenoprasum) (108 mg $100 \mathrm{~g}^{-1}$ de MF), goiabeira-serrana (25,2 mg $100 \mathrm{~g}^{-1}$ de MF), hemerocalis (75,98 mg $\left.100 \mathrm{~g}^{-1} \mathrm{de} \mathrm{MF}\right)$ e cravina (89,78 mg $100 \mathrm{~g}^{-1}$ de MF) (Amarante et al., 2019; Costa, Ribeiro e Barbosa, 2014; Fernandes et al., 2016; Stefaniak \& Grzeszczuk, 2019).
A vitamina $C$ é importante para a manutenção da saúde da pele, gengivas e vasos sanguíneos, além da prevenção do escorbuto, melhora do sistema imunológico humano e é um importante antioxidante (Lee \& Kader, 2000). No entanto, a vitamina C não é produzida pelo organismo humano, necessita ser ingerida, e os alimentos como frutas e hortaliças são as principais fontes desta vitamina. A IDR (ingestão diária recomendada) estabelecida para adultos no Brasil é de $45 \mathrm{mg}$ (Brasil, 2005) e $75 \mathrm{mg}$ pela FAO/ OMS (Fernandes et al., 2016). Desta forma, a ingestão diária de $100 \mathrm{~g}$ de flores de capuchinha, supre totalmente a recomendação de IDR de vitamina C. Mesmo com a redução dos conteúdos de vitamina $\mathrm{C}$ com o armazenamento em temperatura ambiente $\mathrm{e}$ refrigerada a $15^{\circ} \mathrm{C}$, o consumo de $100 \mathrm{~g}$ também supre totalmente a IDR do Brasil e em 73,2\% e 62,4\% a da FAO/OMS, respectivamente, indicando serem fontes importantes desta vitamina na dieta humana, mesmo após oito dias do armazenamento.

\section{Conclusões}

É possível armazenar flores de capuchinha por até oito dias sem perda das qualidades visuais e nutricionais em ambiente refrigerado a $5^{\circ} \mathrm{C}$ e $10^{\circ} \mathrm{C}$.

$\mathrm{O}$ armazenamento das flores de capuchinha em temperatura ambiente não é aconselhável, pois ocorre imediata perda de qualidade.

O uso alimentar de flores de capuchinha é uma boa alternativa na agregação de nutrientes a dieta humana.

\section{Literatura Citada}

AMARANTE, C. V. T. do, et al. 2019. Physicochemical attributes and functional properties of flowers of Brazilian feijoa genotypes. Pesquisa Agropecuária Brasileira 54(445):1-5.

AMMAR, L. et al. 2014. Characterization of two prickly pear species flowers growing in Tunisia at four flowering stage. LWT - Food Science and Technology 59(1):448-454.

ANDRADE JUNIOR, V. C. et al. 2016. Conservação pós-colheita de frutos de morangueiro em diferentes condições de armazenamento. Horticultura Brasileira 34(3):405-411. 
BARRET, D. M.; BEAULIEU, J. C.; SHEWFELT, R. 2010. Color, flavor, texture and nutritional quality of fresh-cut fruits and vegetables: desirable levels, instrumental and sensory measurement, and the effects of processing. Critical reviews in Food Science and Nutrition 50(5):369-389.

BENVENUTI, S.; BORTOLOTTI, E.; MAGGINI, R. 2016. Antioxidant Power, anthocyanin content and organoleptic performance of edible flowers. Scientia Horticulturae 199(1):170-177.

BRASIL. MINISTÉRIO DA SAÚDE. AGÊNCIA NACIONAL DE VIGILÂNCIA SANITÁRIAANVISA. 2005. Aprova o regulamento técnico sobre a ingestão diária recomendada (IDR) de proteína, vitaminas e minerais. Resolução RDC $\mathrm{n}^{\circ} 269$, de 22 de setembro de 2005. Diário Oficial da União, Poder Executivo. Brasília.

CANTERI, M. G. et al. 2001. SASM - Agri: Sistema para análise e separação de médias em experimentos agrícolas pelos métodos Scoft Knott, Tukey e Duncan. Revista Brasileira de Agrocomputação 1(2):18-24.

CHERUT, A. J. 2009. Changes in non-enzymatic antioxidant and ajmalicine production in Catharanthus roseus with different soil salinity regimes. Botany Research International 2(1):1-6.

CHITARRA, M. I. F.; CHITARRA, A. B. 2005. Póscolheita de frutas e hortaliças: fisiologia e manuseio. 2. ed. Lavras, MG, UFLA.785p.

COSTA, L. C. da; RIBEIRO, W. S.; BARBOSA, J. A. 2014. Compostos bioativos e alegações de potencial antioxidante de flores de maracujá, cravo amarelo, rosa e capuchinha. Revista Brasileira de Produtos Agroindustriais 16(6):279-289.

COUTO, M. A. L.; CANNIATTI-BRAZACA, S. G. 2010. Quantificação de vitamina C e capacidade antioxidante de variedades cítricas. Ciência e Tecnologia de Alimentos 30(1):15-19.

FERNANDES, L. et al. 2016. Uma perspectiva nutricional sobre flores comestíveis. Acta Portuguesa de Nutrição 6(1):32-37.

FREDDO, A. R.; CECHIN, F. E.; MAZARO, S. M. 2013. Conservation of post-harvest leaves of green onion (Allium fistulosum L.) with the use of salicylic acid solution. Brazilian Journal of Applied Technology for Agricultural Science (Guarapuava) 6(3):87-93.

INSTITUTO ADOLFO LUTZ. 2008. Métodos físicoquímicos para análise de alimentos. 1. ed. digital. São Paulo. 1020p.

KAISOON, O. et al. 2011. Phenolic compounds and antioxidant activities of edible flowers from Thailand. Journal of Functional Foods 3(2):88-99.

KELEN, M. E. B. et al. 2015. Plantas Alimentícias não convencionais (PANCs): hortaliças espontâneas e nativas. 1 ed. UFRGS, Porto Alegre. 44p.

LEE, S. K.; KADER, A. A. 2000. Preharvest and postharvest factor influencing vitamin $\mathrm{C}$ content of horticultural crops. Postharvest Biology and Technology 20(3):207-220.

LIBERATO, P. da S.; LIMA, D. V. T. de; SILVA, G. M. B. da. 2019. PANCs - Plantas alimentícias não convencionais e seus benefícios nutricionais. Environmental Smoke 2(2):102-111.

LORENZI, H. MATOS, F. J. A. 2007. Plantas medicinais no Brasil: nativas e exóticas. São Paulo, Instituto Plantarum. 2 ed. 576p.

OLIVEIRA, D. S. et al. 2011. Vitamina C, carotenoides, fenólicos totais e atividade antioxidante de goiaba, manga e mamão procedentes da Ceasa do estado de Minas Gerais. Acta Scientiarum-Health Science 33(1):89-98.

OGASSAVARA, F. O. et al. 2009. Comparação entre cultivares de carambola para produção de produtos minimamente processados. Revista Brasileira de Fruticultura 31(2):544-551.

PIETRO, J. et al. 2012. Qualidade de rosas de corte tratadas com produtos naturais. Ciência Rural (Brasil) 42(10):1781-1788.

RIBEIRO, W. S.; BARBOSA, J. A.; COSTA, L. C da. 2012. Capuchinha (Tropaeolum majus L.). Brasília, Editora Kirion. 100p.

SANGALLI, A.; SCALON, S. P. Q.; CARVALHO, J. C. L. 2007. Perda de massa de flores de capuchinha após armazenamento. Horticultura Brasileira 25(3):471-474. 
SANTO, A. P. E. et al. 2007. Efeito anticoagulante in vitro do extrato hidroetanólico de folhas e flores édulas de Tropaeolum majus L. (Tropaeolaceae) sobre o plasma humano. Latinamerican Journal of Pharmacy (Buenos Aires) 26(5):732-736.

SILVA, D. F. da, et al. 2013. Conservação pós-colheita de fisális e desempenho produtivo em condições edafoclimáticas de Minas Gerais. Revista Ceres (Brasil) 60(6):826-832.

SILVA, E. N. da, et al. 2018. Determinação do ponto de colheita de flores de Tropaeolum majus L. Revista Brasileira de Agropecuária Sustentável 8(1):37-43.

SONEGO, G.; BRACKMANN, A. 1995. Conservação pós-colheita de flores. Ciência Rural (Brasil) 25(3):473-479.

SOUZA, A. G. de, et al. 2016. Postharvest quality of feijoa flowers treated with different preservative solutions and 1-Methylcyclopropene. Revista Brasileira de Fruticultura 38(4):e-759.
SOUZA, A. G. de; CARVALHO, J.; ANAMI, J. M. 2018a. Refrigeração e ácido ascórbico na conservação de cebolinha-verde minimamente processada. Revista Agropecuária Catarinense (Brasil) 31(2):58-62.

SOUZA, A. G. de; CARVALHO, J.; ANAMI, J. M. 2018b. Conservação de couve minimamente processada tratada com ácido ascórbico. Revista Evidência (Brasil) 18(2):147-160.

SOUZA, V. C.; LORENZI, H. 2005. Botânica Sistemática: guia ilustrado para identificação das famílias de Angiospermas da flora brasileira. Nova Odessa, SP, Instituto Plantarum. 640p.

SOUZA, A. G. de, et al. 2019. Efeito da refrigeração na conservação de hortaliças orgânicas minimamente processadas. Revista Evidência (Brasil) 19(2):131-148.

STEFANIAK, A.; GRZESZCZUK, M. 2019. Nutritional and biological value of five edible flower species. Notulae Botanicae Horti Agrobotanici (Cluj-Napoca) 47(1):128-134. 